\title{
Über biologische Wirkungen schneller Elektronen eines 6-MeV-Betatrons*
}

\author{
Von Wolfgang Paul und Gerhard Schubert \\ Aus dem II. Physikalischen Institut der Universität Göttingen (Dir.: Prof. Dr. H. K o p f e r mann) \\ und der Universitäts-Frauenklinik Hamburg-Eppendorf (Dir.: Prof. Dr. G. S c h u b e r t)
}

(Z. Naturforschg. 5 b, 390-394 [1950]; eingegangen am 23. Oktober 1950)

\begin{abstract}
Mit Hilfe des Göttinger Betatrons der Siemens-Reiniger-A.-G. wurde der Einfluß schneller Elektronen auf verschiedene biologische Objekte überprüft. Bei der Mehrzahl der bisher untersuchten strahlenbiologischen Reaktionen (Wachstumshemmung von Gerstenkeimlingen, Mitosehäufigkeit in Wurzelspitzenzellen der Gerste und der Pferdebohne, Strahlenabtötung von Drosophila-Eiern und -Puppen u. a.) ist die Wirksamkeit schneller Elektronen je Dosiseinheit geringer als diejenige von Röntgenstrahlen $(200 \mathrm{kV})$. Bei der Bestimmung der strahleninduzierten geschlechtsgebundenen Letalfaktoren bei Drosophila ließ sich kein Unterschied zwischen den beiden Strahlenarten feststellen, gleichgültig, ob es sich dabei um die Bestrahlung reifer oder unreifer Spermien handelt. Das gleiche gilt für das strahleninduzierte Verhalten von einfachen Chromosomenbrüchen bei Drosophila. Nur bei der Strahlenabtötung von Coli-Bakterien sind schnelle Elektronen je Dosiseinheit wirksamer als Röntgenstrahlen. Die Ergebnisse lassen sich treffertheoretisch deuten.
\end{abstract}

$\mathrm{D}_{\mathrm{H}}^{\mathrm{i}}$ Göttinger Elektronenschleuder der SiemensReiniger-A.-G. liefert schnelle Elektronen mit Energien bis zu 6 Millionen Elektronvolt (MeV). Innerhalb dieses Energiebereiches beruht die biologische Wirkung der schnellen Elektronen fast ausschließlich auf der Entstehung von Ionisationen oder Anregungen von Molekülen. Energieverluste der Strahlung durch andere Prozesse, z. B. durch die entstehende Röntgenbremsstrahlung oder durch Atomumwandlungsprozesse, haben quantitativ nur untergeordnete Bedeutung.

Mit Hilfe des Betatrons lassen sich bekanntlich auch ultraharte Röntgenstrahlen herstellen. Trotz grundlegender physikalischer Unterschiede zwischen schnellen Elektronen und ultraharten Röntgenstrahlen dürften die biologischen Wirkungen, die von den beiden Strahlenarten bei gleicher Energie ausgelöst werden, völlig gleichartig sein. Das folgt aus der einfachen Tatsache, daß Röntgenstrahlen ebenfalls nur durch die von ihnen ausgelösten Sekundärelektronen wirksam werden. In diesem Falle ist das Ausmaß der biologischen Wirkung in einem isolierten kleinen Gewebsvolumen weitgehend von der Zahl der pro Masseneinheit durchstrahlter Substanz gebildeten Ionen, also von der Strahlendosis, abhängig.

Unterschiede in der biologischen Wirksamkeit zweier Strahlungen sind jedoch trotz gleicher Dosis dann zu erwarten, wenn sich die beiden Strahlungen wesentlich in ihrer spezifischen Ionisation unterscheiden. Man versteht darunter die Zahl der Ionen, die pro $\mathrm{cm}$ Bahnlänge eines primär oder sekundär entstandenen Teilchens erzeugt werden. Die lineare
Ionendichte ist bei den einzelnen ionisierenden Strahlungen außerordentlich verschieden. Bei der von uns benutzten schnellen Elektronenstrahlung von $6 \mathrm{MeV}$ ist der mittlere Ionenabstand rund 10-mal so groß wie bei Röntgenstrahlen von $200 \mathrm{kV}$. Ein weiterer Unterschied zwischen den beiden Strahlungen beruht auf der verschiedenen Häufigkeit größerer oder kleinerer Ionisationsgruppen. Man erkennt auf der linken Seite der Nebelkammeraufnahme (Abb. 1), daß die Sekundärelektronen von 200-kV-Röntgenstrahlen längs ihrer gekrümmten Bahnen größere Ionisationsgruppen hervorrufen. Bei einer schnellen Elektronenstrahlung liegen die Ionisationen längs der gestreckten Bahn (rechts im Bild) zumeist in kleineren Gruppen, erst am äußersten Ende der Elektronenbahn kommen größere Ionenhaufen zustande.

Unser Hauptaugenmerk galt zunächst der Frage, wie sich diese Absorptionsunterschiede. zwischen schnellen Elektronen und den wesentlich energieärmeren Röntgenstrahlen einer üblichen Therapieanlage für $200 \mathrm{kV}$ auf biologische Objekte auswirken. Von den Colibakterien ist nach den Untersuchungen von Wyckoff, Zirkle, Lea sowie $\mathrm{H}$ a in es und $\mathrm{Brets} \mathrm{cher}{ }^{1}$ bekannt, daß sie schon

1 R. W. G. Wy ck off, J. gen. Physiol. 15, 351 [1932]. - R. E. Z i r k l e, J. gen. comp. Physiol. 16, 221 [1940]. - D. E. Lea, R. B. H a ines u. E. Brets cher, J. Hyg. 41, 1 [1941]. - C. Di e ckmann, W. Dittrich, H. Reich u. G. S chubert, Naturwiss. 37, 140 [1950].

* Auszugsweise vorgetragen von G.S chubert auf dem 6. Internationalen Radiologenkongreß in London am 26 . Juli 1950. 
durch eine einzige primäre Ionisation abgetötet oder in ihrer Teilungsfähigkeit gehemmt werden, falls dieser „leichte“ Treffer innerhalb eines strahlenempfindlichen Bereichs von etwa $12 \mathrm{~m} u$ Durchmesser erfolgt. Wir untersuchten das Verhalten von Bacterium coli comm. gegenüber gleichen Dosen von schnellen Elektronen und Röntgenstrahlen. Wie die Abb. 2 zeigt, steigen die Schädigungskurven für beide Strahlungen nach einer Exponentialfunktion an. Das bedeutet, daß tatsächlich Eintreffervorgänge für die Strahlenabtötung von Colibakterien maßgeblich sind. Die Schädigungskurve für schnelle Elektronen verläuft jedoch steiler als die für Röntgenstrahlen, die

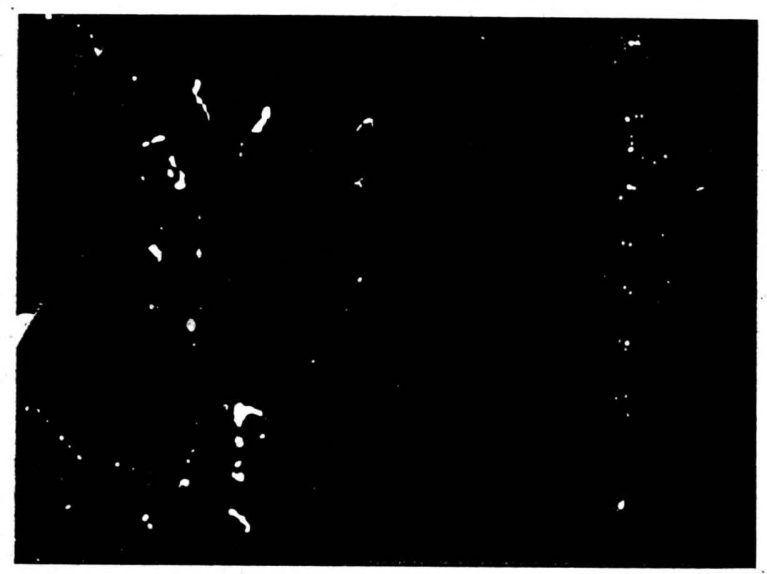

Abb. 1. Nebelkammeraufnahme der Bahnen von Sekundärelektronen einer 200-kV-Röntgenstrahlung (links) und von schnellen Elektronen (rechts).

Halbwertsdosis sinkt mit zunehmendem Ionenabstand ab. Schnelle Elektronen sind hier also je Dosiseinheit wirksamer als Röntgenstrahlen. Dieses Ergebnis läßt sich zwanglos dadurch deuten, daß bei der dichter ionisierenden Röntgen-Strahlung häufiger zwei oder mehrere Ionisationen in einen Treffbereich hineingeraten und dort eine Reaktion auslösen, wozu bereits ein leichter Treffer in Form eines Ionenpaares genügen würde. Die Wirkung der einzelnen Ionisationen wird also - trotz gleicher Ionenzahl je Volumeneinheit Luft - um so besser ausgenützt, je größer ihre mittleren Abstände längs der Bahn des ionisierenden Teilchens sind, wie es z. B. bei den schnellen Elektronen des Betatrons der Fall ist. Die Treffertheorie interpretiert diesen Effekt als Sättigungseffekt.

Es sei hier erwähnt, daß die Dosismessungen bei unseren Elektronenbestrahlungen mit Hilfe von klei- nen dünnwandigen Fingerhutkammern $(0,2 \mathrm{~mm}$ Cellonwand) durchgeführt wurden. Die innen graphitierte Ionisationskammer war an einen Momentandosismesser angeschlossen. Das Meßverfahren wurde durch Benutzung eines biologischen Dosismaßes kontrolliert, nämlich durch die strahleninduzierte $\mathrm{Ab}$ tötungsrate von 2-stdg. Drosophila-Eiern. Diese Reaktion verläuft erfahrungsgemäß unabhängig von der spezifischen Ionisation der angewandten Strahlung. Alle bisherigen Versuche zeigten, daß physikalisch gleiche Dosen von 180-kV-Röntgenstrahlen und schnellen Elektronen auch die gleiche biologische Wirkung ausüben.

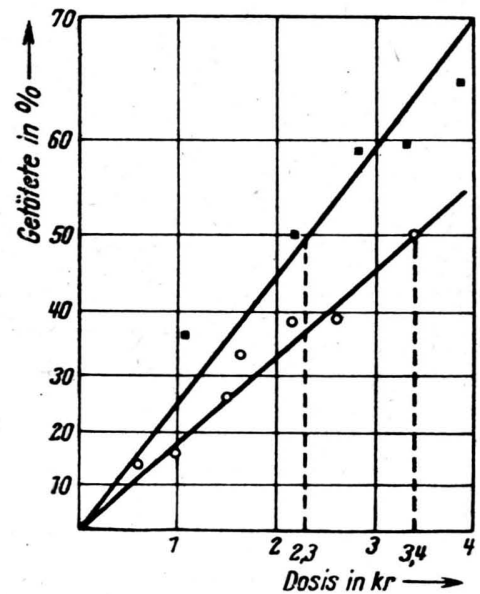

Abb. 2. Tötung von Bact. coli durch schnelle Elektronen und Röntgenstrahlen. $\bigcirc$ Röntgenstrahlen: $180 \mathrm{kV}, 6 \mathrm{~mA}$, HWS 0,27 $\mathrm{mm} \mathrm{Cu}, 500 \mathrm{r} / \mathrm{min}$. - Schnelle Elektronen: $3 \mathrm{MeV}$.

Eine weitere wohlbekannte strahlenbiologische Reaktion ist das strahleninduzierte Auftreten rezessivgeschlechtsgebundener Letalfaktoren in den Spermien der Taufliege Drosophila melanogaster. Nach heutigen Anschauungen tritt der mutagene Effekt dann ein, wenn eine Ionisation innerhalb eines strahlenempfindlichen Volumens in der Größenordnung von etwa 100 bis 2000 Atomen erfolgt. Die Abb. 3 zeigt zunächst (punktierte Linie) den Verlauf der Dosiseffektkurven bei Röntgen- und $\gamma$-Strahlen nach Angaben von $\mathrm{Tim}$ of é eff - R es s ovsky und $\mathrm{Zim}$ mer ${ }^{2}$. Die Mutationsrate ist bei weichen und harten Röntgenstrahlen, bei $\beta$ - und $\gamma$-Strahlen gleich. Auch unsere Kontrolluntersuchungen mit Röntgenstrahlen von $180 \mathrm{kV}$ passen sich den bereits bekannten Werten

2 N. W. Timoféeff-Ressovsky u. K. G. Zimmer, Biophysik I, Das Trefferprinzip in der Biologie. Verlag Hirzel, Leipzig 1947. 


\begin{tabular}{|c|c|c|}
\hline Strahlung & $\begin{array}{c}\text { Reife Spermien } \\
\text { 1-3 Tage nach } \\
\text { Behandlung }\end{array}$ & $\begin{array}{c}\text { Unreife Spermien } \\
21-27 \text { Tage nach } \\
\text { Behandlung }\end{array}$ \\
\hline $\begin{array}{c}\text { Röntgenstrahlen } \\
(180 \mathrm{kV} 3000 \mathrm{r})\end{array}$ & 8,4 & 2,8 \\
$\begin{array}{c}\text { Schnelle Elek- } \\
\text { tronen (3000 r) }\end{array}$ & 9,5 & 2,5 \\
\hline
\end{tabular}

Tab. 1. Geschlechtsgebundene rezessive Letalfaktoren im $\mathrm{X}$-Chromosom (in Prozenten) bei reifen und unreifen Spermien.

gut an. Mit Hilfe der ClB-Methode wurde nun der Einfluß der schnellen Elektronen von $3 \mathrm{MeV}$ auf die Genmutationsrate geprüft ${ }^{3}$. Die Ergebnisse bei insgesamt rund 10000 geprüften Spermien zeigen, daß die Rate der geschlechtsgebundenen Letalmutationen bei Einwirkung schneller Elektronen nur wenig gröBer ist als die röntgeninduzierte Rate. Der Unterschied ist jedoch so wenig signifikant, daß daraus kaum auf einen unterschiedlichen Einfluß von schnellen Elektronen und Röntgenstrahlen auf die Genmutationsrate geschlossen werden kann, wenn man die Fehlerbreite des Meßverfahrens berücksichtigt. Der Verlauf der Dosiseffektkurven ist mit der Annahme einer Eintrefferreaktion vereinbar. Der Treffbereich, das Gen, ist so klein, daß im allgemeinen jeweils nur eine einzige Ionisation in den Treffbereich hineinfällt. In diesem Fall ist der mutagene Effekt einzig und allein von der Ionenzahl je Masseneinheit abhängig. Bei den von uns geprüften Strahlungen war deshalb zu erwarten, daß sie näherungsweise die gleiche biologische Wirkung haben, was durch das Experiment bestätigt wurde.

Das gleiche gilt für die Mutationsrate von Spermien, die sich zur Zeit der Bestrahlung noch im Stadium unreifer Geschlechtszellen befanden. Analog den Arbeiten anderer Autoren ${ }^{4}$ ließ sich feststellen (Tab. 1), daß nach Bestrahlungen mit gleicher Dosis bei reifen Spermien eine höhere Rate von geschlechtsgebundenen Letalfaktoren vorhanden ist als bei unreifen Spermien ${ }^{5}$. Dieser Unterschied ist teilweise durch das Vorhandensein einer ausgesprochenen germinalen Selektion und vielleicht auch durch gewisse Unterschiede der Genmutabilität bedingt. Innerhalb

3 Dittrich, Höhne, Paul u. Schubert, Naturwiss. 1950, im Druck.

4 B. B. H a r r i s, J. Hered. 20, 299 [1929]. - F. B. H a n s on u. F. H e y s, Amer. Naturalist 68, 97 [1934]. - N. W. T i m of é eff - R essovsky, Biol. Zbl. 57, 233 [1938].

5 Dittrich, Höhne, Paul u. Schubert, noch unveröffentlicht. der beiden Gruppen von röntgen- und elektronenbestrahlten Spermien sind aber keine Unterschiede in der biologischen Wirkung der beiden Strahlungen vorhanden.

Von besonderem Interesse erschien uns das strahleninduzierte Verhalten von einfachen Chromosomenbrüchen bei Drosophila melanogaster. Als Testobjekt

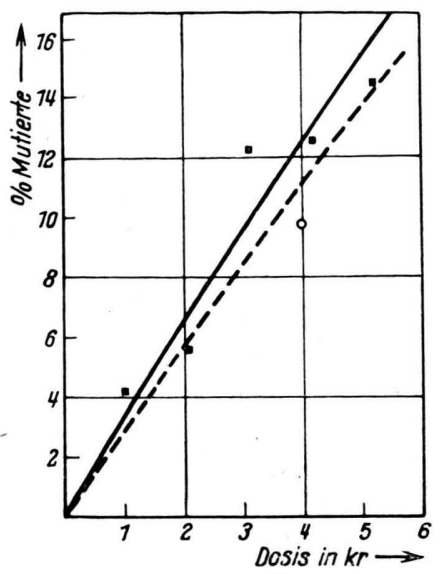

Abb. 3. Rate geschlechtsgebundener rezessiver Letalmutationen nach Bestrahlung mit Röntgenstrahlen (TimoféeffRessovsky) (......) und schnellen Elektronen von $3 \mathrm{MeV}(-\bullet)$.

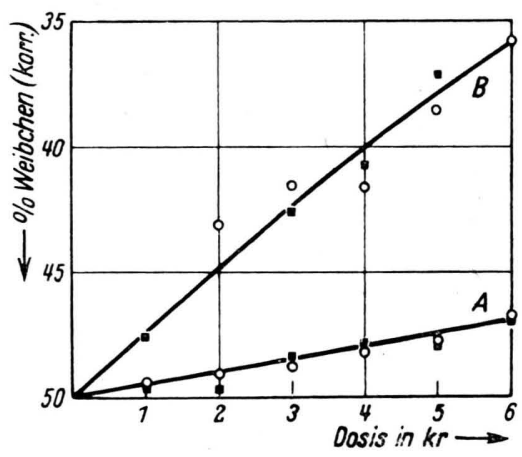

Abb. 4. Prozentsatz der Weibchen in der $F_{1}$-Generation von bestrahlten Männchen eines $\mathrm{X}+$-Stammes (A) und eines Ring-X-Stammes (B). Röntgenstrahlen. - Schnelle Elektronen.

wurde der $\mathrm{X}^{\mathrm{c}^{2}}$-Stamm von Drosophila gewählt. Die Eigenart dieses Stammes beruht darauf, daß die Männchen an Stelle des stäbchenförmigen X-Chromosoms ein ringförmiges X-Chromosom bei sonst gleichartigem Genbestand besitzen. Solche Männchen mit einem Ring-X-Chromosom wurden mit schnellen Elektronen von $3 \mathrm{MeV}$ Energie oder mit $180-\mathrm{kV}$ Röntgenstrahlen bestrahlt und dann mit normalen Weibchen gepaart. Die als Bestrahlungsfolge auftretenden einfachen Chromosomenbrüche rufen Trans- 
lokationen und bizentrische Chromatiden hervor; diese wirken bei den weiblichen Zygoten letal. Sie manifestieren sich also in einer Verschiebung des Geschlechtsverhältnisses in der $\mathrm{F}_{1}$-Generation $\mathrm{zu} \mathrm{Un}$ gunsten der Weibchen. Abb. 4 enthält zunächst (untere Kurve) das Weibchendefizit nach Röntgenoder Elektronenbestrahlung von Spermien mit normalem stäbchenförmigem X-Chromosom. Die verhältnismäßig geringe Herabsetzung der Weibchenziffer ist durch Deletionen und vielleicht auch durch dominante letale Genmutationen bedingt, dabei er-

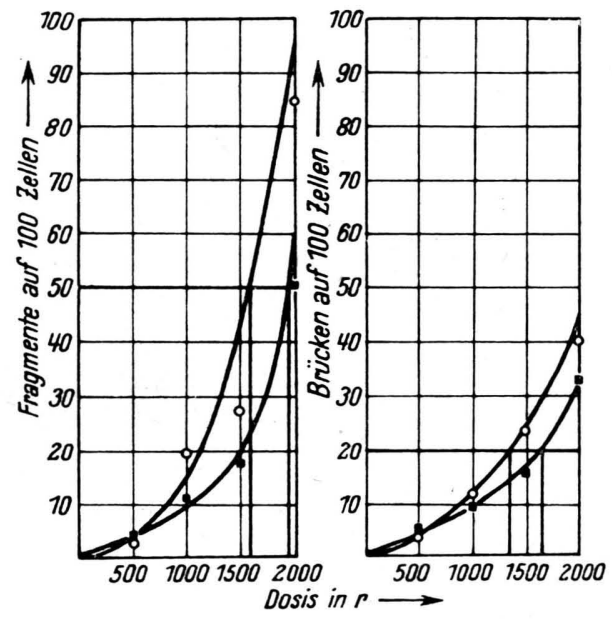

Abb. 5. Häufigkeit der Chromosomenfragmente und Chromosomenbrücken bei Hordeum vulgare (Anaphasen und Telophasen zusammengefaßt). Röntgenstrahlen. - Schnelle Elektronen.

zeugen gleiche Dosen der beiden Strahlungen auch den gleichen Effekt. Werden jedoch Männchen mit einem Ring-X-Chromosom bestrạhlt, dann tritt in der $\mathrm{F}_{1}$-Generation eine stärkere Geschlechtsverschiebung hervor (obere Kurve), und zwar steigt das Weibchendefizit annähernd proportional mit der Dosis an. Die Ergebnisse lassen jedoch keinen gesicherten Unterschied in der Wirkung von Röntgenstrahlen und schnellen Elektronen erkennen. Die strahleninduzierten Chromosomenmutationen von Drosophila verhalten sich in dieser Hinsicht also ebenso wie Genmutationen ${ }^{6}$.

Ganz anders liegen die Verhältnisse, falls zur Auslösung einer Strahlenreaktion ein größerer Energiebetrag, d. h. die rasche Anhäufung einer größeren

6 v. Brandt, Höhne, S chubert u. Zi m m e $r \mathrm{~m}$ a $n \mathrm{n}$, noch unveröffentlicht.

7 H. B l e ek, W. P a u l, H. J. S ch merm und u. G. S chu bert, Naturwiss. 36, 28 [1949]:
Zahl von Ionisationen innerhalb des strahlenempfindlichen Bereichs notwendig ist. Nach den ausgezeichneten Untersuchungen von $\mathrm{L}$ e a sind etwa 15 bis 20 Ionisationen erforderlich, um z. B. einen Chromatidbruch innerhalb eines pflanzlichen Zellkerns hervorzurufen. In diesem Falle müssen die Betatronstrahlungen weniger wirksam sein als die dichter ionisierenden Röntgenstrahlen von $200 \mathrm{kV}$, weil nur Röntgenstrahlen bei gleicher r-Dosis eine entsprechend größere Anzahl von „schwereren“ Treffern aufbringen. Wir untersuchten die Häufigkeit von strahleninduzierten Chromosomenstörungen in Wurzelspitzenzellen von Hordeum vulgare ${ }^{7}$ und Vicia $f a b a^{8}$. Man sieht auf Abb. 5, daß die Zahl der Chromosomenfragmente und der Chromosomenbrücken bei Vergrößerung der Dosis anwächst. Die Wirkungskurven der schnellen Elektronen verlaufen jedoch flacher als die von Röntgenstrahlen $(200 \mathrm{kV})$; das läßt auf den erwarteten geringeren Effekt der schnellen Elektronen bei gleicher r-Dosis schließen. Das gleiche gilt für entsprechende Untersuchungen bei Vicia faba. Für derartige Reaktionen, die stets durch Mehrtrefferprozesse bedingt anzusehen sind, gibt es Dosisbereiche, in denen die Wirkung einer Bestrahlung mit zunehmender spezifischer Ionisation trotz gleicher Ionenzahl je Volumeneinheit größer wird. In diesem Fall summieren sich entweder die einzelnen Ionisierungen im Treffbereich. Oder aber es erhöht sich die Wirkung der einzelnen bei einem Teilchendurchgang entstehenden Ionisierungen nicht einfach additiv, vielmehr nimmt die-Wirkungswahrscheinlichkeit noch stärker zu. J or d a ${ }^{9}{ }^{9}$ bezeichnet diesen Wirkungsanstieg als Konzentrationseffekt.

Ähnlich den vorgenannten Versuchsreihen wurde die Wirkung der schnellen Betatronelektronen auf eine ganze Reihe von möglichst unterschiedlichen Reaktionsformen der lebenden Materie geprüft. Die Tab. 2 gibt einen zusammenfassenden Überblick über unsere Ergebnisse bei den verschiedensten pflanzlichen und tierischen Objekten. In der letzten Spalte der Tabelle ist das Verhältnis der sich auf einen bestimmten biologischen Effekt beziehenden Röntgendosis zur äquivalent wirkenden Elektronendosis angegeben. Man erkennt, daß das Wirkungsverhältnis der beiden Strahlungen von Objekt zu Objekt verschieden ist. Bei Colibakterien ist das Wirkungsverhältnis z. B. ein anderes als bei Vicia faba. Die relative biologische Wirksamkeit variiert auch bei den

\footnotetext{
8 Heinrich, Paul u. Schubert, Naturwiss. 1950, im Druck.

9 P. J or d a n, Naturwiss. 26, 693 [1938].
} 


\begin{tabular}{|c|c|c|c|c|}
\hline Objekt & Reaktion & $\begin{array}{l}\text { Wirksame } \\
\text { Elektronen- } \\
\text { dosis } \\
\text { [in r] } \\
\end{array}$ & $\begin{array}{c}\text { Wirksame } \\
\text { Röntgendosis } \\
\text { [in r] } \\
\end{array}$ & $\begin{array}{l}\text { Röntgen- } \\
\text { dosis/Elek- } \\
\text { tronendosis }\end{array}$ \\
\hline $\begin{array}{l}\text { Bacterium coli comm. . . } \\
\text { Hordeum vulgare }\end{array}$ & Abtötung von $50 \%$ & 2200 & 3200 & 1,45 \\
\hline 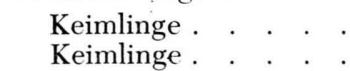 & $\begin{array}{l}\text { Wachstumshemmung von } 10 \% \text { der Keime } \\
\text { Hemmung des Blattlängenwachstums }\end{array}$ & 4800 & 2600 & 0,5 \\
\hline & um $50 \%$ & 3100 & 1700 & 0,6 \\
\hline $\begin{array}{l}\text { Wurzeln . } \cdot \text {. } \\
\text { Wurzelspitzenzellen : }\end{array}$ & $\begin{array}{l}\text { Wachstumshemmung um } 30 \% \\
\text { Chromosomenbrïcken, }\end{array}$ & 3000 & 1500 & 0,5 \\
\hline Wurzelspitzenzellen & $\begin{array}{l}20 \text { in } 100 \text { Mitosezellen } \\
\text { Chromosomenfragmente, }\end{array}$ & 1600 & 1300 & 0,8 \\
\hline Vicia faba & & 1950 & 1600 & 0,8 \\
\hline $\begin{array}{l}\text { Wurzelspitzenzellen } \\
\text { Wurzelspitzenzellen }\end{array}$ & $\begin{array}{l}\text { Pathologische Ana- und Telophasen } 50 \% \\
\text { Chromosomenverkürzung um } 20 \% \%\end{array}$ & $\begin{array}{l}610 \\
360\end{array}$ & $\begin{array}{l}490 \\
230\end{array}$ & $\begin{array}{l}0,8 \\
0,6\end{array}$ \\
\hline Drosophila melanogaster & & & & \\
\hline $\begin{array}{l}\text { Eier } 2 \text { Stdn. alt } \\
\text { Eier } 2^{3 / 4} \text { Stdn. alt . } \\
\text { Eier } 6^{1 / 4} \text { Stdn. alt } \\
\text { Puppen. . . . . }\end{array}$ & $\begin{array}{l}\text { Abtötung von } 50 \% \\
\text { Abtötung von } 50 \% \\
\text { Abtötung von } 50 \% \\
\text { Abtötung von } 50 \%\end{array}$ & $\begin{array}{r}195 \\
1225 \\
1060 \\
3050\end{array}$ & $\begin{array}{r}205 \\
460 \\
825 \\
2800\end{array}$ & $\begin{array}{l}1,0 \\
0,4 \\
0,8 \\
0,9\end{array}$ \\
\hline $\begin{array}{l}\text { Imagines } \\
\text { Reife Spermien . } \\
\text { Unreife Spermien } \\
\text { Spermien . . . } \\
\text { Spermien . . . . }\end{array}$ & $\begin{array}{c}\text { Rezessive letale Genmutationen } \\
10 \% \text { im X-Chromosom } \\
\text { Rezessive letale Genmutationen } \\
2 \% \text { im X-Chromosom } \\
\text { Dominante Chromosomenmutationen } \\
20 \% \text { im Ring-X-Chromosom } \\
\text { Dominante Chromosomenmutationen } \\
6 \% \text { im X+-Chromosom }\end{array}$ & $\begin{array}{r}\sim 3200 \\
\sim \\
\sim \\
4000 \\
4000 \\
6000\end{array}$ & $\begin{array}{r}\sim 3500 \\
\sim 2200 \\
4000 \\
6000\end{array}$ & $\begin{array}{l}\sim 1 \\
\sim 1 \\
\quad 1,0 \\
1,0\end{array}$ \\
\hline
\end{tabular}

Tab. 2. Relative biologische Wirksamkeit schneller Elektronen im Vergleich zu Röntgenstrahlen.

verschiedenen Strahlenreaktionen innerhalb des gleichen Objekts. Bei Gerstenwurzeln ist das Wirkungsverhältnis bezüglich der Auslösung von Chromosomenfragmenten größer als hinsichtlich des Hemmungseffektes des Wurzelwachstums. Auch bei der gleichen Strahlenreaktion, z. B. der Abtötung von Drosophila-Eiern, sind Wirkungsänderungen vorhanden, wenn sich das biologische Material in einem Zustand anderer Stoffwechselaktivität befindet. Zusammenfassend läßt sich feststellen, daß die relative biologische Wirksamkeit schneller Elektronen im Vergleich zu Röntgenstrahlen $(200 \mathrm{kV})$ innerhalb weiter Grenzen schwanken kann. Bei der Mehrzahl der geprüften biologischen Reaktionen sind schnelle Elektronen jedoch weniger wirksam als Röntgenstrahlen. Dabei handelt es sich vorzugsweise um solche Re- aktionen, die eine größere Anzahl von Ionisationen, also „schwerere“ Treffer innerhalb eines strahlenempfindlichen Bereichs, erfordern. Dagegen werden solche Reaktionen, die schon durch die Wirkung "leichter" Treffer ausgelöst werden, quantitativ begünstigt bei den Betatronstrahlungen auftreten. Dieses bei verschiedenartigen biologischen Objekten und Reaktionen gewonnene Ergebnis muß sich ohne weiteres auf ein einheitliches biologisches Objekt übertragen lassen, in welchem mehrere strahlenbiologische Reaktionen nebeneinander und gleichzeitig ablaufen. Das würde aber nichts anderes bedeuten, als daß neben den quantitativen auch qualitative Unterschiede in der biologischen Wirkung von eingestrahlten schnellen Elektronen und Röntgenstrahlen mittlerer Energie vorhanden sein können. 